\title{
EVIDENCE BASED NURSING INTERVENTIONS IN PREVENTION OF PRESSURE ULCER AMONG CHILDREN
}

\author{
P.Sumathy, S. Rajeswari*
}

\begin{abstract}
Pressure ulcer (PU) has now become a common problem among the Paediatric population. The risk factors can be classified into intrinsic and extrinsic factors. There are several scales for assessment of pressure ulcer for children but the Braden Q scale is found to be more valid and reliable, and for the new born, it is the Neonatal Skin Risk Assessment Scale (NSRAS). All children who are admitted should have a comprehensive assessment and those with pressure injuries are staged as per National Pressure Ulcer Advisory Panel. Nurses have got a pivotal role in prevention of pressure ulcer among children by adopting various preventive strategies.
\end{abstract}

Key Words : Pressure Ulcer, Braden Q scale, Neonatal Skin Risk Assessment Scale

\section{Introduction}

Excellent skin care is an attribute of quality care. Prevalence of skin breakdown and pressure ulcer has become a standard by which hospitals are evaluated and assessed with pressure ulcers recognized as an international patient safety problem. Most pressure injuries are preventable, if appropriate measures are implemented. Clinical practice guidelines for prevention and treatment of pressure ulcers that specifically address the needs of the pediatric population. ${ }^{1}$

\section{Pressure Ulcer Prevalence Rates}

While the problem of Pressure ulcers in adults has received a great deal of attention, far less is known about pressure ulcer in children and neonates. 1 Recent studies have indicated that PUs are also common in the pediatric population, and in the last ten years greater attention has been paid to this problem. There is greater awareness that pediatric patients in certain health care settings are also at high risk of developing Pressure ulcers. Prevalence rates for PUs in hospitalized pediatric patients range from $3 \%$ to $28 \%{ }^{2}$ The pressure ulcer prevalence rates are as high as $27 \%$ in PICU and $23 \%$ in NICU, most of it which occurs within 2 days of admission. ${ }^{1}$

\section{Risk factors for Pressure Ulcers}

Pressure ulcers are defined as a localized area of tissue destruction that develops as a result of soft tissue being compressed between a bony prominence and an external surface, causing starvation of oxygen and vital nutrients. ${ }^{3}$

A pressure ulcer can develop in as short as 30 minutes if there is a high pressure in a small area, increased pressure over short periods of time and slight pressure for long periods of time has shown to cause equal damage. Several factors have been identified to cause skin breakdown in the pediatric population. Although the true risk factors are difficult to determine there are certain suggested risk factors which can be classified into intrinsic and extrinsic factors. ${ }^{4}$

* Prof. Mrs. P. Sumathy, Mrs. S. Rajeswari, Asso .Prof, Dept. of Child Health Nursing, KGNC, Puducherry 607402, India. . 
Extrinsic Factors - Impact Injury loss, Heat, Moisture, Posture, Infection.

Intrinsic Factors - Immobility, Sensory loss, Age Disease, Body type , Incontinence, Poor Nutrition.

Both the factors are related to Pressure Ulcer

\section{Who are the Children at Risk:}

Neonates

$>$ Length of stay $>4$ days

$>$ Edema

$>$ Weight loss

$>$ Sepsis

$>$ Traction devices

$>$ Mechanical ventilator

$>$ Children with spina bifida and cerebral palsy

$>$ Extra corporeal membrane oxygenator

$>$ Duration of intubation

$>$ Medical devices. ${ }^{5}$

\section{Skin Differences:}

- The difference in the skin of an infant from that of an adult predisposes the infant to a higher risk of skin injury because of a lack of healthy and mature skin barrier. ${ }^{6}$

- The infant skin has also has a higher absorption rate as compared with an adult's skin. This difference in the absorption rate also predisposes infants to a dry, flaky and impaired skin barrier. Moreover the skin cells are smaller and thinner than an adult skin, which results in a weakened barrier to the environment. ${ }^{7}$

- A preterm infant's skin is also prone to injury because of the lack of collagen in the skin. Collagen helps to maintain the strength and the elasticity of the dermis and hence if deficient, leads to a higher risk of an injury to the skin. ${ }^{8}$

\section{Risk Assessment scales for Pressure Ulcer in Pediatric Population:}

Although there is no agreement on which risk factors contribute to pressure ulcer development in neonates and children. There is an agreement that prevention lies in early identification. ${ }^{9}$ There are around 10 published pediatric pressure ulcer risk assessment scales, out of which the Braden Q scale for predicting pediatric pressure ulcer risk is a widely used valid and reliable pediatric specific pressure ulcer risk assessment tool which is adapted from the adult based Braden scale. ${ }^{10}$ The sensitivity of the Braden Q scale was found to be $88 \%$ and a specificity of $58 \% .{ }^{11}$ The Braden Q scale includes the 6 original Braden subscales (mobility, activity, sensory perception, moisture, friction and shear, and nutrition) and in addition has a 7th component i.e., tissue perfusion /oxygenation.

Braden Q Scale can be used for children $<5$ years , and Adult Braden Scale is used for children $>5$ years. $^{12}$

\section{Scoring the Braden Q Scale}

The total Braden $\mathrm{Q}$ scale scores range from 7 (highest risk ) to 28 (lowest risk), with a score of 16 or lower identifying pediatric patients at risk for pressure ulcers. ${ }^{11}$

The other assessment scales include the Glamorgan scale and the Neonatal Skin Risk Assessment Scale (NSRAS), which has got a sensitivity of $98.4 \%$, and $83 \%$ and a specificity of $67.5 \% \& 81 \%$ respectively. ${ }^{13}$

\section{Patient and Wound Assessment:}

On admission all neonates and children should have a comprehensive assessment that includes skin assessment and risk assessment for pressure ulcers.

Skin assessment: Accurate head to toe skin assessment should be carried out which includes visual inspection of the anterior and posterior surface of the skin. Thorough examination of high risk areas such as under splints, braces, traction boots, tracheostomy plates and arm boards is critical. ${ }^{14}$

The risk assessment is carried out by using the Risk Assessment Scales.(Braden Q Scale, Glamorgan Scale, Neonatal Skin Risk Assessment Scale (NSRAS).

If pressure ulcers are noted location, size, undermining , tunneling, drainage, necrotic tissue, epithelisation of any stage and surrounding skin tissue should be documented. Pressure injuries are staged according to the 2009 National Pressure Ulcer Advisory Panel and European Pressure Ulcer Advisory Panel (NPUAP/EUPAP), Injury classification System. ${ }^{15}$ 


\section{Stage 1 : Pressure Injury Non-Blanchable Erythema:}

- Intact skin with non blanchable redness of a localized area usually over a bony prominence

- The area may be painful, firm, soft, warmer or cooler compared to adjacent tissue.
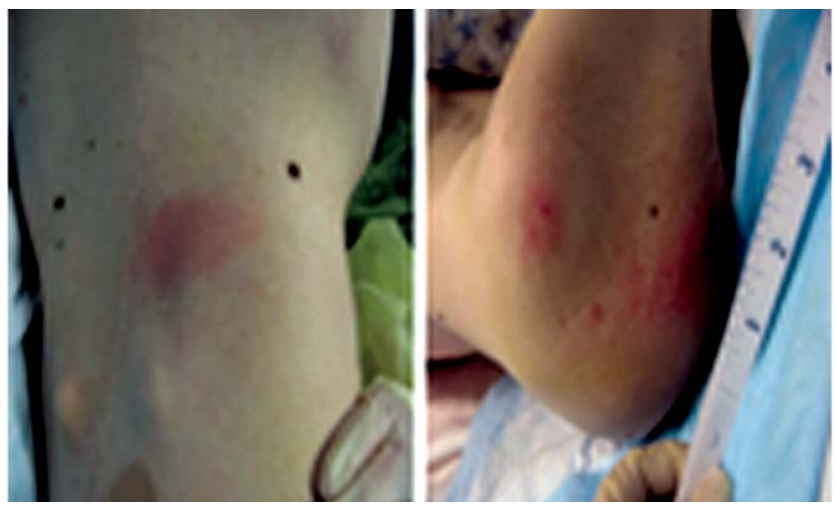

- Darkly pigmented skin may not have visible blanching, its colour may be different from the surrounding area.

- May indicate at risk persons (heralding sign of risk)

\section{Stage 2 Pressure Injury : Partial Thickness Skin Loss}
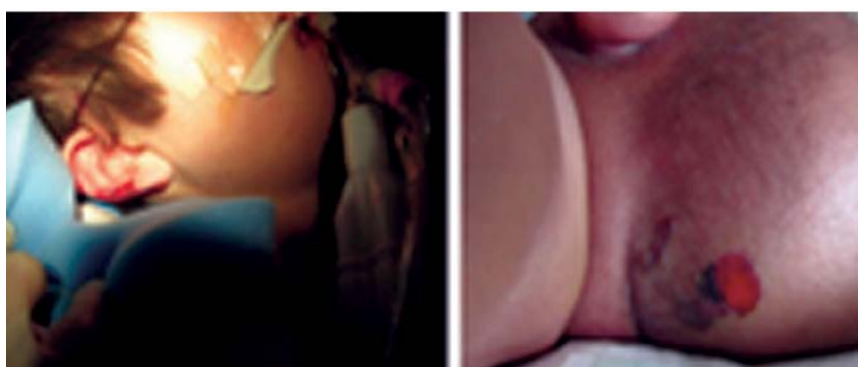

- Presents as a shallow, open wound with a red pink wound bed, without slough.

- May also present as an intact or open/ruptured serum filled blister.

- Presents as a shiny or dry, shallow ulcer without slough or bruising.

\section{Stage 3 Pressure injury: Full Thickness} Skin Loss
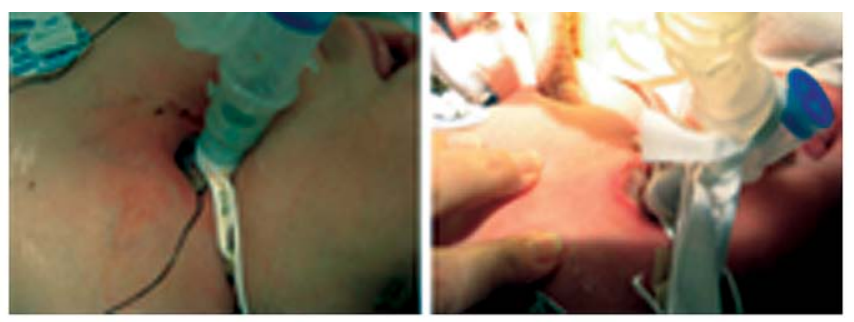

- Subcutaneous fat may be visible but bone, tendon muscle are not exposed. Slough may be present but does not obscure the depth of tissue loss. May include tunneling and undermining.

- The bridge of the nose, ear, occiput and malleolus do not have subcutaneous tissue and can be shallow, but areas of significant adiposity can develop extreme deep injuries.

\section{Stage 4 Pressure Injury: Full Thickness Tissue Loss}

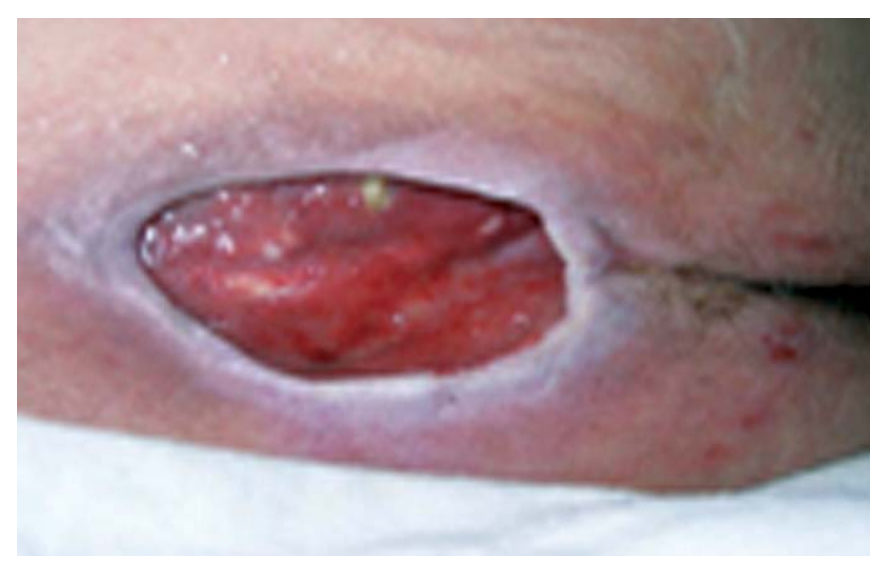

Full thickness full tissue loss with exposed bone, tendon or muscle. Slough or eschar may be present on some parts of the wound bed.

The depth of a stage 4 pressure injury varies by anatomical location. It can extend into muscles and /or supporting structures, making osteomyelitis possible. Exposed bone or tendon is visible or directly palpable.

\section{Unstageable Pressure Injury : Depth Unknown}

Full thickness tissue loss in which the base of the pressure injury is covered by slough (yellow, tan, grey, 
green or brown ) and or eschar (tan, brown or black) in the pressure injury bed.

Until enough slough/eschar is removed to expose the base of the pressure injury, the true depth and therefore the stage, cannot be determined. Stable (dry, adherent, intact without erythema or fluctuance) eschar on the heels serves as the body's natural biological cover and should not be removed.

\section{Evidence Based Interventions for Prevention and Treatment of Pressure Ulcer - Key Role of the Skin Champion's - The Nurses}

Prevention of pressure ulcer and early detection is the goal of all health care providers. Nurses play an important role in the prevention of pressure ulcers. Hence it is essential to develop strategies to prevent the occurrence. ${ }^{14}$

The strategies include:

$\checkmark$ Increase awareness of pressure ulcer risk.

$\checkmark$ Identify, assess, and monitor children at moderate and high risk.

$\checkmark \quad$ Initiate pressure ulcer prevention protocol

$\checkmark$ Interdisciplinary education

$\checkmark$ Pressure ulcer data collection (weekly skin audits)

$\checkmark$ Make recommendations for pressure ulcer prevention and treatment. ${ }^{5}$

\section{Prevention and Treatment of Pressure Ulcer: Interventions should aim at}

1. Positioning the child

2. Minimizing or eliminating friction and shear/ minimizing pressure

3. Managing moisture

4. Maintaining adequate nutrition/hydration

5. Educating mothers /caregiver

1.Positioning: Turning and re-positioning schedule every 2 hours. The aim of repositioning is to reduce or eliminate pressure in order to maintain circulation to areas of the body at risk for pressure ulcer development. ${ }^{16}$

2. Minimize pressure: The occipital area is the most common anatomical site in children for the development of pressure injury, followed by the sacrum, ear lobes and heels. ${ }^{14}$
- The risk of developing pressure ulcer in the occipital area can be done by changing the position of the head and repositioning the patients every $2^{\text {nd }}$ hourly.

- Protective devices such as Gel Pillow, ${ }^{17}$ Foam

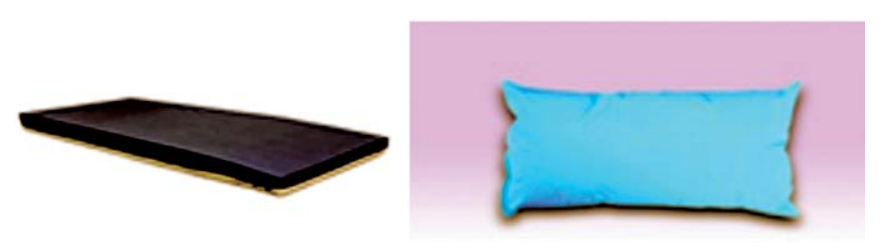

Pillow, Air Fluidized Beds, and Viscous Fluid Mattress are found to be useful In removing the Pressure Off from the Occipital Region. A foam overlay with and without a gel pillow provides an effective and cost effective pressure reducing surface in pediatric patients ages infant through 16 years of age. ${ }^{18}$ Foam mattresses aim to redistribute body weight and the movement of a child is only slightly limited.$^{19}$

- Protection of the heels can be accomplished by suspending the heels off the bed using pillows, gel foams, positional protective pillows or a foam padded boot.

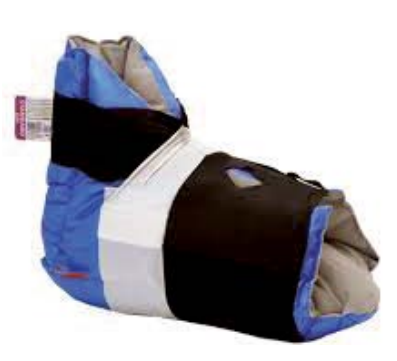

Eg., Prevalon boot - this boot will protect the heel, the lateral ankle and the medial ankle from injury. ${ }^{1}$

- Protective barrier dressing on the sacral region not only includes foam cushioning for the protection of bony prominences but also should protect the skin from shearing with their removal.

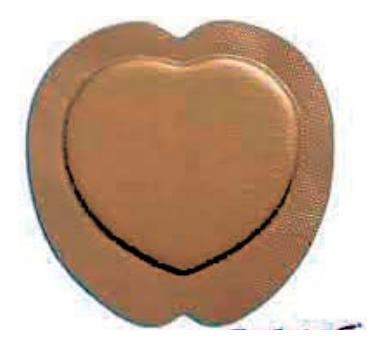

Eg., Mepilex Border Sacrum - this is a silicone based product that is non adherent and is gentle on the skin when removed. ${ }^{20}$ 


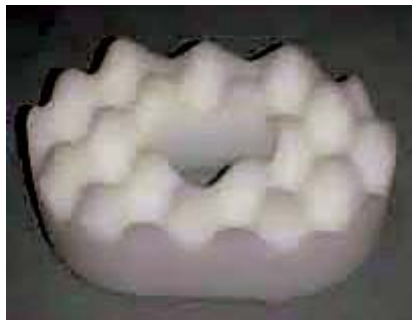

- Ear protector-donut shaped, convoluted polyurethane foam raises the ear from the bed allowing air passage, drainage and protection from bed sores.

Device related pressure injury: Device related injuries are common pressure injuries in children. More than $50 \%$ of the pressure injuries are due to devices. ${ }^{1}$ The common device related injuries includes the use of pulse oximeter, naso gastric tube, CPAP, nasal cannula, and tracheostomy plates. ${ }^{20}$

\section{Strategies to prevent pressure ulcer due to devices}

- Change the location of the pulse oximeter.

- Fenestrated contact dressing can be applied underneath devices such as a pulse oximeter.

- Foam protective barrier dressing can cushion and protect the skin from the pressure of the gastrointestinal tube and absorb any drainage or leakage which may occur from or around the gastrointestinal tube site.

- Silicion tape or a thin hydrocolloid can be beneficial in maintaining the skin integrity to secure devices such as nasal cannulae, prongs etc., ${ }^{1}$

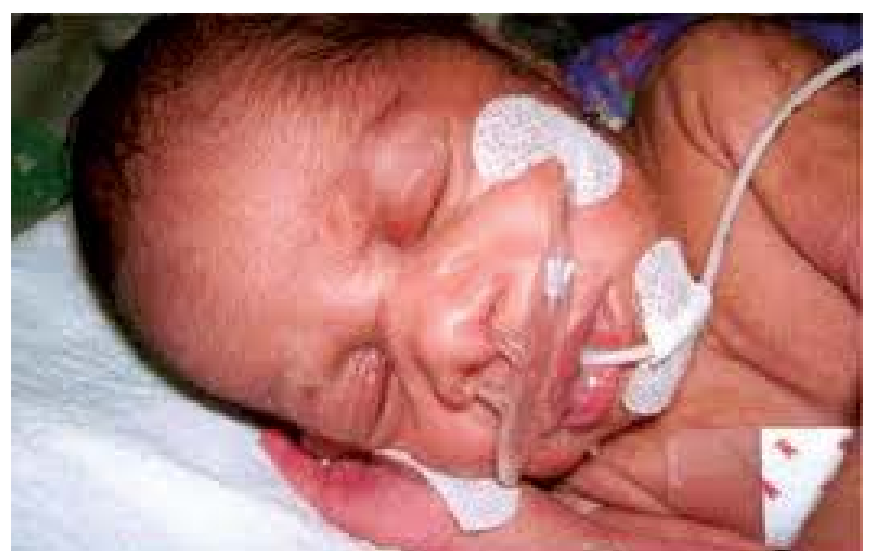

3.Managing of moisture: A moist environment due to faecal or urinary incontinence can cause skin breakdown in the diaper area. A petroleum based ointment or a zinc oxide paste to the skin with each diaper change can be beneficial. ${ }^{21}$

4. Maintain adequate nutrition and hydration: The systemic and immunologic effects of malnutrition further limit the tissue tolerance to pressure, frictional forces and shear especially as third spacing from hypoalbuminemia develops. ${ }^{22} \mathrm{Hence}$, a comprehensive assessment and good nutrition can help prevent injury from occurring. If pressure ulcers have occurred additional proteins, calories, vitamins and minerals are required to promote the wound healing process. ${ }^{20}$

\section{The need for Education:}

Patient education is an important piece of pressure ulcer prevention and treatment. The patient, family and care givers are key to prevention, management and treatment of pressure ulcers. Teaching materials should be given to the patient and family on admission or at the time risk is identified. The areas for education includes causes of pressure ulcers, ways to prevent them, dietary needs, positioning, signs of infection, types of tissue, normal and abnormal colours of tissue, infection control, dressing change technique etc., ${ }^{21}$

\section{Treatment of Pressure Ulcers:}

1. Wound Cleansing: Only sterile water or normal saline is used and most preferred for neonates is sterile water. Normal saline to be diluted with sterile water in the ratio of $1: 1 .^{23}$

2. Debridement: Necrotic tissue should be debrided, but adequate guidelines for managing heel pressure ulcers in neonatal and pediatric population is lacking.

3. Dressings: Recommendations for non infected wounds include the use of hydrogels, hydrocolloids and film dressings. For infected wounds sheet hydrogels can be combined with topical antibacterial and antifungal ointments but must be changed every 6-8 hours ${ }^{24}$

4. Pain management: Researchers have examined the use of topical medications for pressure ulcer pain treatment. ${ }^{25}$ 


\section{Conclusion}

Pressure ulcer prevention in the pediatric patient is accomplished through pressure ulcer awareness with education to all health care providers and families involved in the care of a child. A thorough skin assessment and utilizing a risk assessment guide is essential to determine the patients who are at risk. The successful implementation of new nursing interventions is the key to a comprehensive pressure ulcer prevention program

\section{References}

1. Mona Mylene Baharestani, Catherine R. Ratliff. Pressure ulcers in Neonates and Children.Advances in Skin and Wound Care 2007; 20(4): 208-219.

2. Kottner J, Wilborn D, Dassen T. (2010). Frequency of Pressure ulcers in the Pediatric Population: a Literature Review and new empirical data. International Journal of Nursing Studies; 47: 1330-1340.

3. Bryant R (2006) Acute and Chronic Wounds. Nursing Management. Second edition. Mosby, st louis MO.

4. Willock J, Hughes, Tickle S, Rossiter G, Johnson C, Pye.H. Pressure sores in children- the actual hospital perspective. Journal of Tissue Viability 2000; 10(2):59-62.

5. Rebecca Kraus , Paula Balestrieri, Mary Bolhuis Rebekah Brunoehler, Mary Charter, Wendi Redfern.Pressure Ulcer Prevention Team Wisconson Children's hospital.

6. Schindler C. A., Mikhailov T. A., Fischern K., Lukasiewicz G., Kuhn E. M., Duncan L. (2007). Skin integrity in critically ill and injured children. American Journal of Critical Care, 16 (6), 568-574

7. Blume-Peytavi U., Hauser M., Stamatas G. N., Pathirana D., Graciana Bartels N. (2012). Skin care practices for newborns and infants: Review of the clinical evidence for best practices. Pediatric Dermatology, 29 (1), 1-14.

8. Turnage Carrier .C, Mc Lane KM, Gregurich MA. Interface Pressure Comparison of Healthy Premature infants with various Neonatal Bed Surfaces. Advances in neonatal Care 2008; 8(3): 176-184.

9. Sims A, McDonald R An overview of pediatric pressure care . Journal of Tissue Viability 2003;13:144-148.

10. Catherine Noonan, Sandy Quigley, Martha A.Q, Curley. Using the Braden Q scale to Predict Presure Ulcer risk in
Paediatric Patients ; Journal of Paediatric Nurisng 2011; 26 :566-575.

11. Curley MA, Quigley. Pressure Ulcers in Paediatric Intensive care, Incidence and Associated factors, Pediatric Critical Care Medicine 2003; 4 : 284-90.

12. Quigley.S \&Curley M. Skin integrity in the Pediatric Population -Preventing and Managing Pressure ulcers. Journal of Pediatric Nursing 1996;1: 17-18.

13. Willock J Antony D, Baharestani M. Development of Glamorgan Pediatric Ulcer risk Assessment scale. Nursing Times 2007.

14. Butler C.T. Pediatric skin care: Guidelines for Assessment, Prevention and Treatment. Dermatology Nursing 2007; 19(5):471-485

15. Black J Baharestani, Cuddigan J .National Pressure Ulcer Advisory Panel Updated Staging System. Advanced Skin Wound care.2009.

16. Hardy , Harrell.D Tran K, Smith S,Zins B et. al., Exploring the effects of wound dressing and patient positioning on skin integrity in a pediatric burn facility Ostomy Wound Management 2007; 53(6): 67.

17. Reddy, M., Gill, S. S., \& Rochon, P. A. Preventing pressure ulcers: A systematic review. JAMA: Journal of the American Medical Association2006; 296(8): 974.

18. Mc Lane KM, Krowskop TA, Mc Cord S, Fraley JK. Comparison of interface Pressure in the Pediatric Population among various Support Surfaces. Journal of Wound Ostomy Continence Nurse 2002; 29: 242-51.

19. Parnham A .Pressure ulcer risk assessment and prevention in children. Nursing Children and Young People 2012; 24: 24-29.

20. Schober Flores. C Epidermolysis bullosa-Wound Care Pearls for the Non-infected and Infected Wound. Journal of the Dermatology Nurses Association 2009; (1):21-28.

21. Cakmak S.K. Gul.U, Ozer.S, Yegit Z, Gonu.M Risk factors for Pressure Ulcer. Advances in Skin and Wound Care 2009; 22(9):412-415.

22. Baranoski S, Ayello EA. Wound care Essentials, practice principles, 2nd edition, Philadelphia P.A Lippincott Williams \&Wilkins 2007.

23. Samaniego I Developing a Skin Care Pathway for Pediatrics .Dermatology Nursing 2002;14:393-396.

24. Lund $\mathrm{C}$ Prevention and Management of Skin Breakdown, Nurse Clinic North America 1999;34:907-920.

25. Prentice WM, Roth LJ, Kelly P.Topical Benzydamine Cream and the relief of Pressure Pain. Palliat Med.2004;18(6):520524 